Inflammation

\section{The mediators of steroid action}

\section{from R. J. Flower}

IN the clinic, steroids are widely used for the treatment of many types of inflammatory disease. Although steroids interrupt the inflammatory response at many levels, it is not known precisely how they accomplish this and they can have serious side effects. On page 77 of this issue ${ }^{1}$, Barbara Wallner and her colleagues at Biogen report an important step in dissecting the mechanisms of steroid action - they have succeeded in identifying the human complementary DNA for lipocortin, an endogenous anti-inflammatory substance, whose production is regulated by circulating steroids and is intimately linked to their mechanism of action.

The lipocortins ${ }^{2}$ are a family of related proteins, independently discovered by several groups ${ }^{3-8}$, with the unusual property of blocking the enzyme phospholipase $A_{2}$. This enzyme is important for several reasons. First, it is involved in the signal transduction process, which enables leukocytes to respond to noxious stimuli, causing them to migrate in chemotactic gradients and to effect a repertoire of responses crucial to the development of inflammation and other host defence mechanisms.

Second, by catabolizing membrane phosphatides, phospholipase $\mathbf{A}_{2}$ liberates much of the substrate used for the generation of inflammatory mediators such as the prostaglandins, leukotrienes and platelet activating factor. Preparations containing lipocortin can suppress the production of mediators by cells, prevent neutrophil and macrophage chemotaxis and suppress inflammation itself in animal models. Although the proteins of the lipocortin family have been fairly well characterized biologically, and even highly purified in small amounts, Wallner et al. ${ }^{1}$ present the first structural data.

The authors first purified lipocortin from rat peritoneal lavage fluid (a rich source of this protein) and used the amino-acid sequence information from

\section{0 years ago}

M. Pasteur, at the last sitting of the Paris Academy of Sciences, stated that out of 325 cases of inoculation for hydrophobia, only one had failed - namely, that of the youth Pelletier, who came too long after being bitten, and under very unfavourable conditions. He advocated the establishment of an international hospital, to which patients would come from al parts of the world; and he suggested a discussion as to the locality and the fund for its support. Prof. Pasteur announced that he should next investigate whether diphtheria could not be treated by a similar process.

From Nature 33 423, 4 March 1886. the tryptic fragments obtained to synthesize oligonucleotide probes which were found to hybridize with messenger RNA extracts from both rat and human cells. One of these probes recovers from a human lymphoma-derived cDNA library a DNA sequence that encodes a protein containing the sequence of all the tryptic fragments of rat lipocortin. This gene was expressed in Escherichia coli and the protein thus obtained was found to be a monomer of relative molecular mass $\left(M_{\mathrm{r}}\right)$ about 37,000 , corresponding closely to a commonly observed species of $M_{\mathrm{r}} 40,000$ (the discrepancy could be caused by the absence of glycosylation: a potential glycosylation site has been identified).

Lipocortin has several notable features. The first is the highly polar nature of the molecule: almost a third of its amino acids are charged and these seem to be distributed throughout the length of the molecule. This fact is perhaps suprising. The expectation of most workers was that the protein would be mainly hydrophobic as might befit a membrane-associated protein involved in the control of lipid metabolism. Another feature is the high degree of homology and immunological crossreactivity which exists between the human and the rat protein, suggesting that this molecule is highly conserved.

Wallner et al. ${ }^{1}$ have examined the occurrence of lipocortin mRNA in the rat and find it widely distributed but most abundant in macrophages, lung, thymus and spleen. These findings correlate quite well with the previously observed distribution of anti-phospholipase activity.

As one might expect, treatment of rats with dexamethasone, a synthetic steroid, led to a rapid increase in lipocortin mRNA in peritoneal macrophages (treatment of these cells in vitro works just as well), although there seems to be a slight anomaly in that in at least one set of experiments this was not followed by the expected rise in cell lipocortin levels.

The inhibitory activity of lipocortin on pancreatic phosphoplipase $A_{2}$ was tested in vitro on a substrate of autoclaved $E$. coli prelabelled with fatty acids. The recombinant material is extremely active in this system, and comparable to the naturally

The blood of all healthy mammals contains circulating glucocorticoid steroids and the toe of the dose-response curve of steroid-stimulated lipocortin release lies within this physiological range. It is therefore not surprising that lipocortin is present in normal cells and tissues. There has been a growing realization among workers occurring protein. in the lipocortin field that this protein is not simply involved in the "crisis management' of inflammation, but almost certainly regulates the activity of membranebound phospolipases under resting conditions as well.

To do this effectively, there needs to be a mechanism whereby phospholipase can be relieved of lipocortin inhibition at times when its activation is essential to bring about a cellular response. One way in which this might occur has been suggested by Hirata's work on lipocortin phosphorylation ${ }^{6}$. He has demonstrated that this protein can be phosphorylated by protein kinases and that this results in a loss of its inhibitory activity which can be subsequently reversed by treatment of the protein with phosphatases. As so many stimuli seem to exert their actions by interacting with cell-surface receptors and causing activiation of protein kinases, this mechanism would seem to be of great topical interest. Wallner et al. ${ }^{1}$ find that lipocortin has two potential phosphorylation sites (on tyrosine and threonine residues) which would fit nicely with Hirata's notion, although it is not yet clear whether phosphorylation leads to inactivation of the recombinant molecule.

The significance of the paper from Wallner et al. lies not so much with the sequencing and cloning of lipocortin as such but in the many avenues now open to us. We obviously want to know the minimum effective inhibitory sequence of lipocortin and as much as possible about the way in which the protein inhibits phospholipase so that drugs can be designed that have the same effect.

The wide spectrum of side effects preclude the use of steroids for long periods of time except in the very seriously ill. These side effects are caused by changes in the transcription of specific genes in the same way as the anti-inflammatory effects. It has long been an article of faith of scientists working in this area that if we could identify and isolate the 'second messengers' of steroid action which are responsible for the anti-inflammatory effects, we should also be able to produce drugs which possess many of the beneficial actions of steroids without incurring the heavy penalty of side effects. The real value of the work by the Biogen team is that it enables us to make headway in that direction.

1. Wallner, B.P.et al. Nature 320, 77 (1986)

Di Rosa, M. et al Prostaglandins 28, 441 (1984)

. Flower, R.J. \& Blackwell, G.J. Nature 278, 456 (1979)

4. Blackwell, G.J. et al. Nature. 287147 (1980).

5. Hirata, F. et al. Proc. natn. Acad. Sci. U.S.A. 77, 2533 (1980).

6. Hirata, F. J. biol. Chem. 256, 7730 (1981).

7. Russo-Marie, F. \& Duval, D. Biochim. biophys. Acta 712, 177 (1982).

8. Cloix, J.F. et al. Br. J. Pharmac. 79, 313 (1983)

R.J. Flower is Professor of Pharmacology in the School of Pharmacy and Pharmacology, University of Bath, Claverton Down, Bath BA2 $7 A Y, U K$ 